\title{
MODELAGEM FUZZY PARA AVALIAÇÃO DA PRODUÇÃO DE MUDAS DE TOMATE CEREJA UTILIZANDO DIFERENTES DOSES DE POLÍMEROS E NÍVEIS DE IRRIGAÇÃO
}

Daniel dos Santos Viais Neto ${ }^{1}$, Valter Alves Pradela ${ }^{2}$, Luís Roberto Almeida Gabriel Filho ${ }^{3}$, Camila Pires Cremasco $^{3}$, Ana Carolina Geraldo de Maria ${ }^{4}$, Gustavo Silva De Oliveira ${ }^{4}$

${ }^{1}$ Faculdade de Tecnologia - FATEC, Presidente Prudente, SP. ${ }^{2}$ Universidade do Oeste Paulista - UNOESTE, Doutorado em Agronomia, Presidente Prudente, SP e Escola Técnica Estadual - ETEC, Teodoro Sampaio, SP. ${ }^{3}$ Universidade Estadual Paulista - UNESP, Faculdade de Ciências e Engenharia, Tupã, SP. ${ }^{4}$ Faculdade de Tecnologia - FATEC, Tecnólogo em Agronegócio, Presidente Prudente, SP

\section{RESUMO}

O tomateiro é considerado a segunda hortaliça de importância econômica no Brasil, e para o sucesso do seu cultivo depende em grande parte da utilização de mudas de qualidade, pois uma muda bem formada, dará origem a uma planta com alto potencial produtivo. A disponibilidade adequada de água é importante para se produzir boas mudas, mas em sua falta, é possível utilizar polímeros comerciais inseridos no substrato. Objetivou-se com este trabalho estudar os efeitos no desenvolvimento das mudas de tomate cereja em diferentes doses de polímeros aplicados no substrato e diferentes níveis de irrigação até a fase de transplantio utilizando modelagem fuzzy. Para tal estudo, utilizou-se de dados de um experimento realizado na Faculdade de Tecnologia de Presidente Prudente. As variáveis de entrada do sistema baseado em regras fuzzy desenvolvido foram Polímero e Irrigação, e para ambas foram definidos 5 conjuntos fuzzy. As variáveis de saída foram comprimento da parte aérea (CPA), comprimento da raiz (CR) e massa seca da muda (MSM). Para as variáveis de saída do SBRF, também foram definidos 5 conjuntos fuzzy. A análise dos efeitos das doses de polímeros e dos níveis de irrigação sobre as mudas de tomate foi realizada por meio de gráficos tridimensionais e mapas de contorno das variáveis de saída. Tal análise, permitiu concluir que, para níveis de irrigação inferiores ao máximo (irrigação diária), a utilização de 2 gramas de polímeros remete às melhores condições das variáveis comprimento da parte aérea, comprimento da raiz e massa seca da muda.

Palavras-chave: lógica fuzzy; massa seca; tomateiro.

\section{FUZZY MODELING FOR EVALUATION OF THE PRODUCTION OF CHERRY TOMATO CHIPS USING DIFFERENT POLYMER DOSES AND IRRIGATION LEVELS}

\begin{abstract}
The tomato is considered the second vegetable of economic importance in Brazil and for the success of its cultivation, depends to a large extent on the use of quality seedlings, since a well formed seedling will give rise to a plant with high productive potential. Adequate water availability is important to produce good seedlings, but failing that, commercial polymers inserted into the substrate can be used. The objective of this work was to study the effects on the development of cherry tomatoes in different doses of polymers applied to the substrate and different levels of irrigation until the transplant phase using fuzzy modeling. For this study, we used data from an experiment conducted at the Faculty of Technology of Presidente Prudente. The input variables of the developed system based on fuzzy rules were Polymer and Irrigation, and for both were defined 5 fuzzy sets. The output variables were shoot length (CPA), root length (CR) and seedling dry mass (MSM). For the SBRF output variables, 5 fuzzy sets were also defined. The analysis of the effects of polymer doses and irrigation levels on tomato seedlings was performed using three - dimensional graphs and contour maps of the output variables. This analysis allowed us to conclude that, for irrigation levels below the maximum (daily irrigation), the use of 2 grams of polymers refers to the best conditions of the variables shoot length, root length and dry mass of the seedling.
\end{abstract}

Keywords: fuzzy logic; dry mass; tomato. 


\section{INTRODUÇÃO}

O tomateiro é considerado a segunda hortaliça de importância econômica no Brasil, sendo em função disso, explorado nas mais diversas regiões, no entanto, para que os rendimentos sejam ótimos, esta cultura tem requerimentos específicos quanto às condições climáticas (ALVARENGA, 2004). O grupo cereja destaca-se pelo seu alto valor comercial e ampla aceitação pelo consumidor, pois esse grupo apresenta muitas variedades regionais com boa tolerância a doenças foliares e pragas (SOUZA, 2003).

O sucesso do cultivo de hortaliças depende em grande parte da utilização de mudas de alta qualidade, o que torna o cultivo de hortaliças mais competitivo, com o aumento de produtividade e diminuição dos riscos de produção (MINAMI, 1995).

A base da horticultura moderna é a produção de mudas de alta qualidade, pois uma muda bem formada dará origem a uma planta com alto potencial produtivo. Para produtores de mudas, ocorre a tendência de se comercializarem mudas mais novas, para reduzir o tempo destas no viveiro de produção (SEABRA JÚNIOR et al., 2004). Os mesmos autores ainda afirmam que, os produtores que irão cultivar estas mudas, preferem mais desenvolvidas, e acredita que essa preferência está relacionada com a facilidade de transplante, pois estas apresentam um sistema radicular compacto, estruturado com um torrão que não se quebra no momento da retirada das bandejas

A disponibilidade adequada de água e nutrientes no substrato é necessária para o bom desenvolvimento de mudas, inclusive de mudas de tomateiro, pois tal hortaliça, é uma das mais exigentes em água, sendo suscetível ao déficit hídrico. O manejo da irrigação permite decidir quando e quanto de água aplicar nas culturas para aperfeiçoar a produção agrícola e para reduzir o consumo desnecessário de água (ALBUQUERQUE; ANDRADE, 2001).

Os polímeros hidrorretentores ou gel agrícola são de origem natural (derivados do amido) ou sintético (derivado do petróleo), valorizados por sua capacidade de absorver e armazenar água (MORAES, 2001), e podem alcançar 400 vezes, ou mais, do seu peso (VALE et al., 2006). Estes polímeros além de reconhecidos pela capacidade de absorção e retenção da água gravitacional na rizosfera (LECIEJEWSKI, 2009), possuem propriedades de melhoria nas características físicas dos solos (VALE et al., 2006). Contudo, é necessário adotar critérios em relação ao uso dos polímeros quanto à dose do produto comercial que será utilizada e, principalmente, à definição do intervalo de irrigação, pois a interação dos polímeros com o substrato utilizado varia de acordo com as características do ambiente de cultivo e com a necessidade das mudas (CARVALHO et al., 2013).

Tais critérios podem ser estabelecidos com a modelagem fuzzy, que consiste neste caso na criação de superfície de ajuste para cada variável aferida, possibilitando assim determinar com exatidão da dose ideal do polímero e também o melhor nível de irrigação.

Nas ciências agrárias, diversas aplicações da lógica fuzzy são estabelecidas buscando-se a criação destes modelos matemáticos, tais como na avaliação do desenvolvimento e da produtividade do tomate híbrido em diferentes tensões de água no solo e diferentes doses de salinidade na irrigação ao longo de seu ciclo (VIAIS NETO, 2016), avaliação do desenvolvimento da cultura da alface, quando irrigada com água tratada magneticamente e convencional (PUTTI, 2015) e avaliação do faturamento do consumo de energia elétrica e demanda de potência ativa e reativa em uma empresa de avicultura de postura (CREMASCO et al., 2010).

Face ao exposto, este trabalho tem por objetivo estudar os efeitos no desenvolvimento das mudas de tomate cereja de diferentes doses de polímeros aplicados no substrato com níveis de irrigação até a fase de transplantio utilizando modelagem fuzzy.

\section{MATERIAL E MÉTODOS Descrição do experimento}

O experimento foi realizado em uma estufa construída com plástico de espessura de 100 micras, medindo $1,9 \mathrm{~m}$ de comprimento por $2,8 \mathrm{~m}$ de largura, com área de $5,3 \mathrm{~m}^{2}$, latitude $22^{\circ} 08^{\prime} 24.7^{\prime \prime}$ Sul e longitude $51^{\circ} 23^{\prime} 04.7^{\prime \prime}$ Oeste. A temperatura no interior da estufa não foi controlada.

Para produção de mudas, foram utilizadas sementes de tomate da variedade cereja, que tem por características, diâmetros variando entre 3 e $10 \mathrm{~cm}$ e formato esférico ou elipsoidal. Nas bandejas, utilizou-se o substrato Bioplant ${ }^{\circledR}$, cuja principal matéria é a casca de pinus, acrescido de várias doses de polímeros Hidrogel ${ }^{\circledR}$. 
Foram consideradas no presente experimento as seguintes variações:

- 1 litro de substrato sem adição de polímero;

- 1 litro de substrato com adição de $1 \mathrm{~g}$ de polímero;

- 1 litro de substrato com adição de $2 \mathrm{~g}$ de polímero;

- 1 litro de substrato com adição de $3 g$ de polímero;

Figura 1. (a) Medição do substrato, (b) pesagem do polímero e (c) mistura do polímero ao substrato.

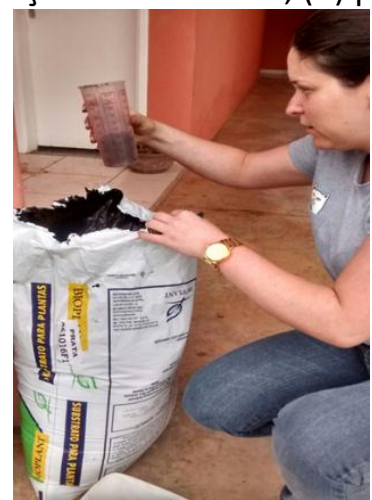

(a)

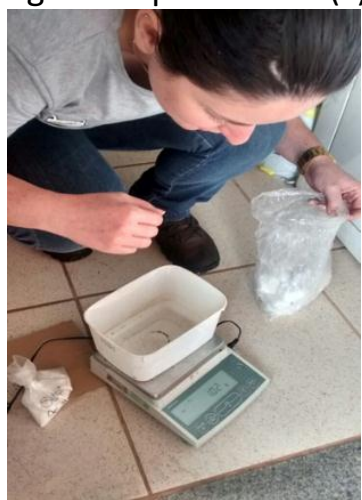

(b)

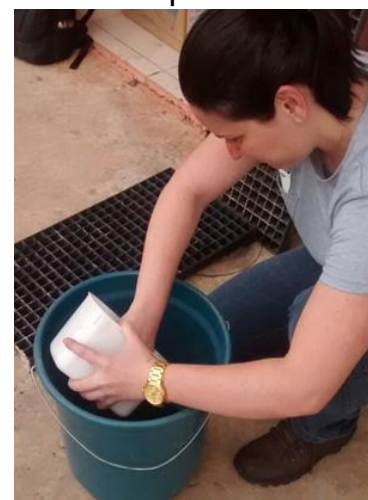

(c)

Fonte: Maria e Oliveira (2017).

A semeadura foi realizada em março de 2017, utilizando-se 3 sementes por célula. A germinação das sementes começou a ocorrer a partir do quinto dia, e após todas terem germinado, foi realizado o desbaste das amostras, restando apenas uma muda por célula. Ao todo foram produzidas 60 mudas para cada dose de polímero aplicada, totalizando no final 300 mudas.

Até este momento, todas as mudas foram irrigadas com água a vontade, depois foi alterada para $4 \mathrm{ml}$ de água, divididos em $1,5 \mathrm{ml}$ ao meio dia e $2,5 \mathrm{ml}$ a seis horas da tarde.

Todos as 5 doses de polímeros supracitados foram submetidas à níveis de
- 1 litro de substrato com adição de 4g de polímero.

Utilizou-se um copo de medida para aferir a quantidade de substrato e uma balança de precisão para pesar o polímero, e antes de misturar o polímero ao substrato, diluiu-se o mesmo em $150 \mathrm{ml}$ de água morna.

A Figura 1 ilustra os procedimentos executados para realizar as misturas.

Figura 2. Identificação dos tratamentos no experimento.

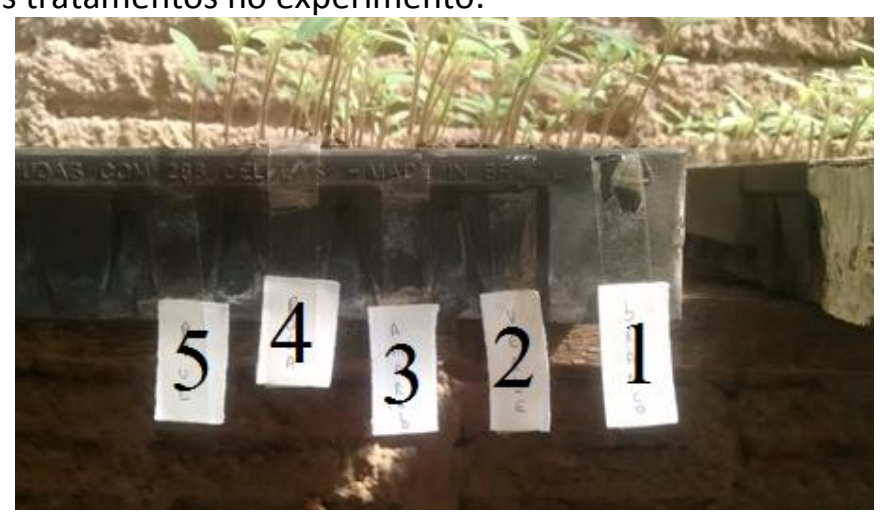

Fonte: Adaptado de Maria e Oliveira (2017). 
Além disto, com tal identificação numérica, associou-se quantitativamente este número com a quantidade de irrigação, sendo tal associação dada por: Irrigação muito alta (5), Irrigação alta (4), Irrigação média (3), Irrigação baixa (2) e Irrigação muito baixa (1).

Após 20 dias da semeadura, período de transplantio utilizado neste trabalho, todas as mudas foram retiradas da bandeja e lavadas com água corrente com 0 intuito de retirar 0 substrato. Depois foram aferidas em relação ao comprimento da parte aérea e da raiz com uso de uma régua. Posteriormente, essas mudas foram acondicionadas em sacos de papel, para que ocorresse a desidratação do material, afim de se obter o peso da massa seca das mudas.

O delineamento experimental adotado foi em blocos inteiramente casualizado, no esquema fatorial $(5 \times 5)$, considerando-se 5 doses de polímeros e 5 níveis de irrigação, com doze repetições, totalizando 300 mudas $(5 \times 5 \times 12)$.

\section{Modelagem fuzzy}

A partir do experimento descrito em Maria e Oliveira (2017), que se utilizou do manejo de diferentes doses de polímeros no substrato (Polímero) e diferentes taxas de irrigação (Irrigação) durante a produção de mudas de tomate cereja, realizou-se uma modelagem fuzzy para estudar os efeitos nas variáveis biométricas no período de transplantio das mudas para o canteiro. As variáveis analisadas foram o comprimento da parte aérea (CPA), o comprimento da raiz (CR) e a massa seca da muda (MSM).
Para tanto, foi considerado o seguinte modelo agronômico para representar a situação proposta: $\quad F: X_{1} \times X_{2} \subseteq \mathbb{R}^{2} \rightarrow \mathbb{R}^{3},\left(x_{1}, x_{2}\right) \mapsto$ $Y=F\left(x_{1}, x_{2}\right)$, onde $X_{1}=[0,4], X_{2}=[1,5], \mathbb{R}$ é o conjunto dos números reais, $x_{1}$ é a quantidade de polímero adicionada no substrato $(\mathrm{g})$ e $x_{2}$ o nível de irrigação utilizado durante desenvolvimento da muda. Os extremos dos intervalos $X_{1}$ e $X_{2}$ estão de acordo com os valores mínimos e máximos utilizados no experimento. Além disso, $Y=F\left(x_{1}, x_{2}\right)=$ $\left(y_{1}, y_{2}, y_{3}\right)$ foi definido pelas medianas das variáveis biométricas da muda do tomateiro, sendo $y_{1} \circ$ comprimento da parte aérea, $y_{2} \circ$ comprimento da raiz, e $y_{3}$ a massa seca da muda. Vale ressaltar que o tratamento Polímero 0 e Irrigação 3 teve sua amostragem comprometida, sendo necessário seu total descarte. Uma vez que seria necessária a mediana de tais dados para ser utilizada como valor representativo de tal tratamento, foi adotada a metodologia de utilizar a média das medianas dos tratamentos do mesmo polímero (Polímero 0) com irrigação inferior (Irrigação 2) e superior (Irrigação 4) à atual, ou seja, foi calculado a média das medianas das aferições dos referidos tratamentos.

Para elaborar um sistema baseado em regras fuzzy (SBRF), foi necessário definir um processador de entrada (ou fuzzyficador), um conjunto de regras linguísticas, um método de inferência fuzzy e um processador de saída (ou defuzzyficador), para no final, gerar um número real como saída (Figura 3). 
Figura 3. SBRF de avaliação das variáveis biométricas das mudas de tomate cereja com 2 variáveis de entrada e 3 variáveis de saída.

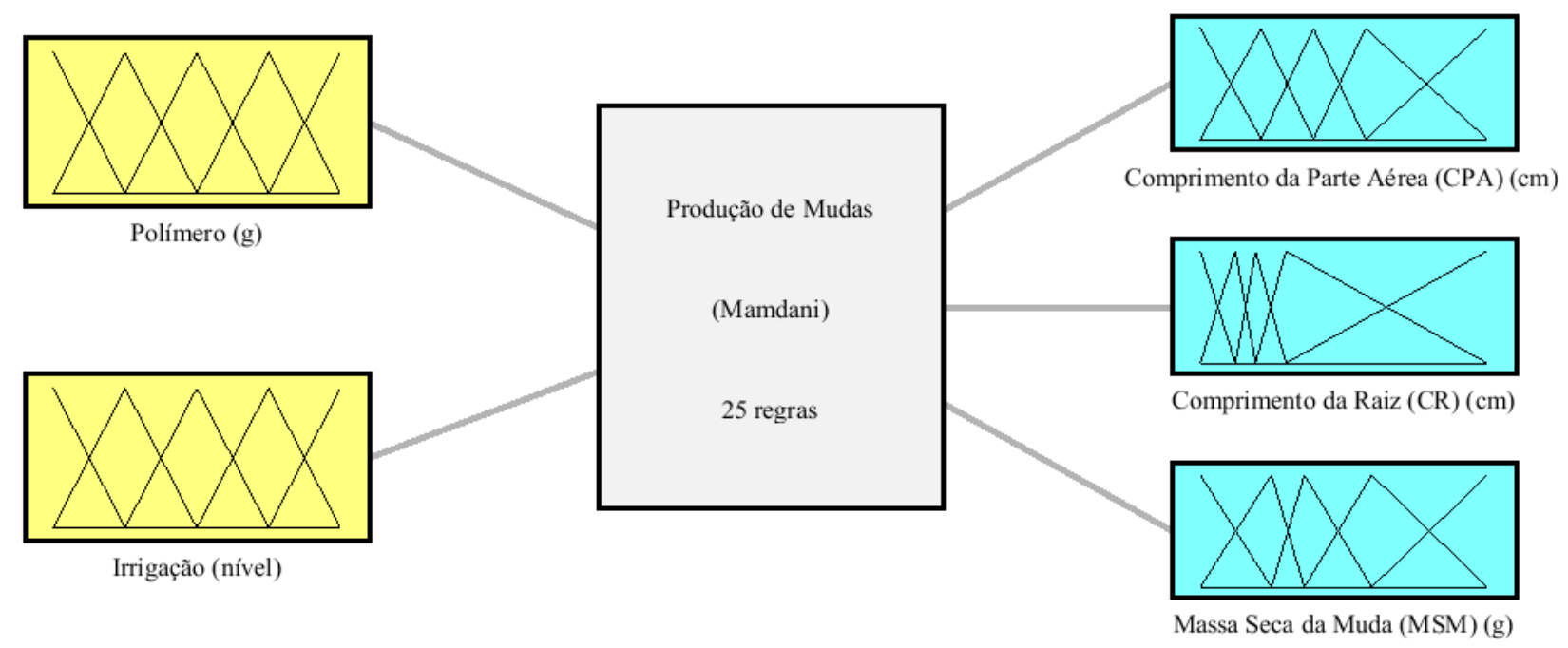

\section{Conjuntos fuzzy das variáveis de entrada}

As variáveis de entrada do SBRF desenvolvido, foram Polímero (g) e Irrigação (níveis de irrigação). Para ambas variáveis, foram definidos 5 conjuntos fuzzy denominados Muito Baixo (MB), Baixo (B), Médio (M), Alto (A) e Muito
Alto (MA) de acordo com as Tabela 1, respectivamente, cujos valores numéricos apresentados foram retirados da análise dos dados do experimento realizado em Maria e Oliveira (2017).

Tabela 1. Definição das funções de pertinência das variáveis de entrada.

\begin{tabular}{cccc}
\hline Conjuntos fuzzy & Tipo & $\begin{array}{c}\text { Delimitadores } \\
\text { Polímero }\end{array}$ & $\begin{array}{c}\text { Delimitadores } \\
\text { Irrigação }\end{array}$ \\
\hline MB & Triangular & {$[-1,0,1]$} & {$[0,1,2]$} \\
B & Triangular & {$[0,1,2]$} & {$[1,2,3]$} \\
M & Triangular & {$[1,2,3]$} & {$[2,3,4]$} \\
A & Triangular & {$[2,3,4]$} & {$[3,4,5]$} \\
MA & Triangular & {$[3,4,5]$} & {$[4,5,6]$} \\
\hline
\end{tabular}

As funções de pertinência das variáveis de entrada são apresentadas na Figura 4.

Figura 4. Funções de pertinência das variáveis de entrada (a) Polímero e (b) Irrigação.

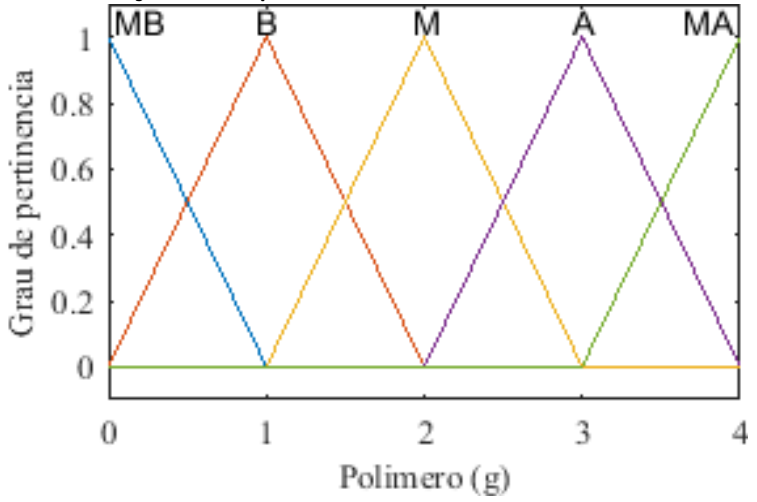

(a)

\section{Conjuntos fuzzy das variáveis de saída}

As variáveis de saída são as seguintes variáveis biométricas da muda de tomate cereja

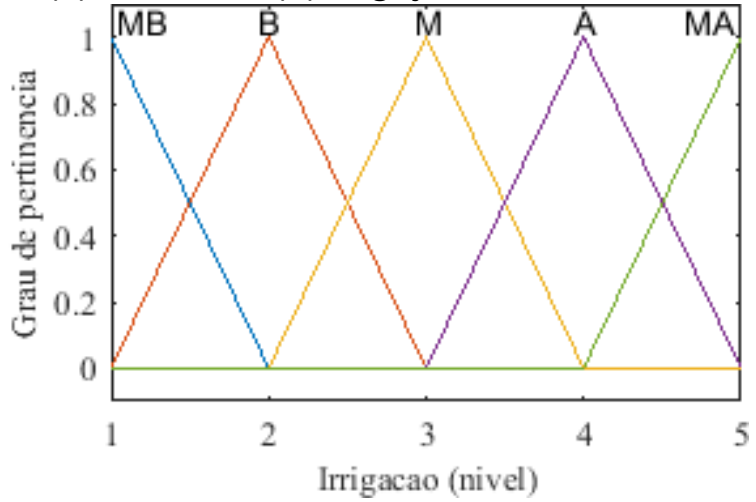

(b)

no período de transplantio da bandeja para o canteiro: comprimento da parte aérea $(\mathrm{cm})$, o 
comprimento da raiz $(\mathrm{cm})$ e a massa seca da muda (g).

Para as variáveis de saída do SBRF foram definidos 5 conjuntos fuzzy semelhante aos utilizados nas variáveis de entrada. Porém, foi necessário criar delimitadores que possibilitassem definir de forma triangular cada uma das funções de pertinência de cada um desses conjuntos fuzzy em questão. Os cálculos a seguir para a determinação de tais delimitadores são semelhantes aos procedimentos metodológicos adotados em Cremasco (2008), Putti (2015), Gabriel Filho (2015) e Viais Neto (2016).
Neste caso, para gerar as funções de pertinência triangulares, calculou-se 5 delimitadores em forma de percentis do conjunto de dados aferidos de cada variável de saída. Estes percentis em $x \%$, denotados por $P(x \%)$, dependem de uma constante $k$, uma vez que os 5 delimitadores necessários são da forma $P(m k)$, $0 \leq m \leq 4$. A Tabela 2 apresenta a definição das funções de pertinência para as variáveis de saída utilizado os percentis variando em $k=25 \%$ $\left(4 k=100 \% \Rightarrow k=\frac{100 \%}{4} \Rightarrow k=25 \%\right)$.

Tabela 2. Definição das funções de pertinência das variáveis de saída.

\begin{tabular}{ccc}
\hline Conjuntos fuzzy & Tipo & Delimitadores \\
\hline $\mathrm{MB}$ & Triangular & {$[\mathrm{P}(0 \%)-(\mathrm{P}(25 \%)-\mathrm{P}(0 \%)), \mathrm{P}(0 \%), \mathrm{P}(25 \%)]$} \\
$\mathrm{B}$ & Triangular & {$[\mathrm{P}(0 \%), \mathrm{P}(25 \%), \mathrm{P}(50 \%)]$} \\
$\mathrm{M}$ & Triangular & {$[\mathrm{P}(25 \%), \mathrm{P}(50 \%), \mathrm{P}(75 \%)]$} \\
$\mathrm{A}$ & Triangular & {$[\mathrm{P}(50 \%), \mathrm{P}(75 \%), \mathrm{P}(100 \%)]$} \\
$\mathrm{MA}$ & Triangular & {$[\mathrm{P}(75 \%), \mathrm{P}(100 \%), \mathrm{P}(100 \%)+(\mathrm{P}(100 \%)-\mathrm{P}(75 \%))]$} \\
\hline
\end{tabular}

\section{Base de regras}

Para a obtenção da base de regras, considerou-se as $25(5 \times 5)$ combinações entre os conjuntos fuzzy das duas variáveis de entrada. Assim criou-se 25 pares da forma Polímero $x$ Irrigação conforme metodologia desenvolvida em Cremasco (2008), Gabriel Filho et al. (2011), Putti (2015), Gabriel Filho (2015) e Viais Neto (2016).

\section{Método de inferência e defuzzyficação}

O método de inferência utilizado neste trabalho foi o Método de Mamdani proposto por Mamdani e Assilian (1975). Já na defuzzyficação, foi utilizado o Método do Centro de Gravidade, que pode ser compreendido como uma média ponderada (CREMASCO, 2008).

\section{Softwares utilizados}

Para a elaboração do SBRF, foram utilizados planilhas eletrônicas e o software Matlab $^{\circledast}$, cuja licença para seu uso a Faculdade de Ciências e Engenharia - FCE/UNESP, Campus de
Tupã - SP, possui. Além disso, utilizou-se a ferramenta Fuzzy Logic Toolbox do software Matlab ${ }^{\circledR}$, para determinar as funções de pertinência, os gráficos tridimensionais e os mapas de contorno do SBRF.

\section{RESULTADOS E DISCUSSÃO}

Neste tópico, apresentou-se inicialmente os resultados obtidos em relação à modelagem $\mathrm{e}$ posteriormente, os resultados sobre os efeitos das doses de polímeros e níveis variados de irrigação na produção de mudas de tomate cereja.

\section{Sistema baseado em regras fuzzy}

Com a determinação dos percentis dos dados aferidos (Tabela 3) no experimento agronômico realizado por Maria e Oliveira (2017) e utilizando a metodologia proposta, foram elaboradas as funções de pertinência dos conjuntos fuzzy das variáveis de saída do presente trabalho (Figura 5), nomeadamente. 
Tabela 3. Valores de percentis dos dados utilizados para avaliar a produção de mudas de tomate cereja por meio de um SBRF.

\begin{tabular}{cccc}
\hline Percentil (\%) & CPA & CR & MSM \\
\hline $0 \%$ & 1,5 & 2,25 & 0,004 \\
$25 \%$ & 5 & 4 & 0,018 \\
$50 \%$ & 8 & 5 & 0,024 \\
$75 \%$ & 11 & 6,5 & 0,037 \\
$100 \%$ & 18 & 16,5 & 0,059 \\
\hline
\end{tabular}

Figura 5. Funções de pertinência das variáveis de saída (a) Comprimento da parte aérea, (b) Comprimento da raiz e (c) Massa seca da muda.

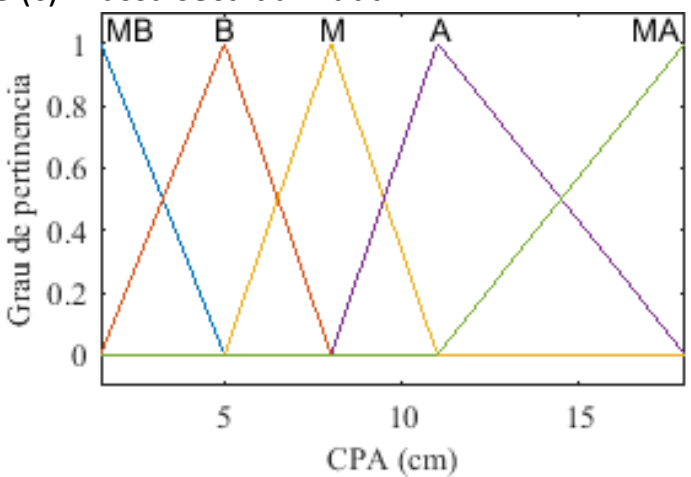

(a)

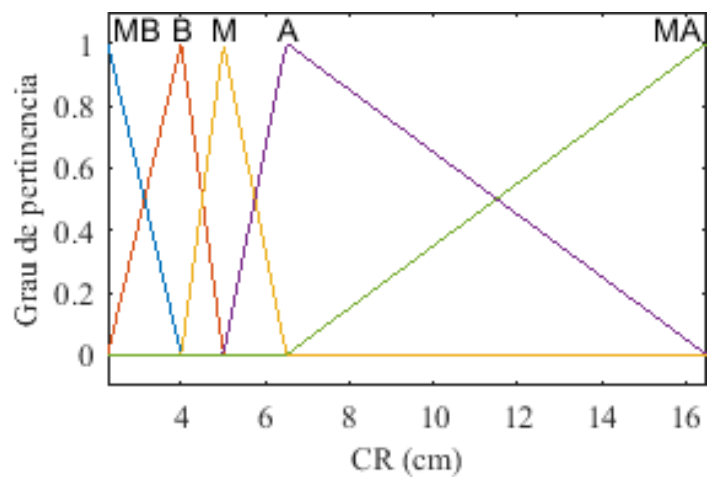

(b)

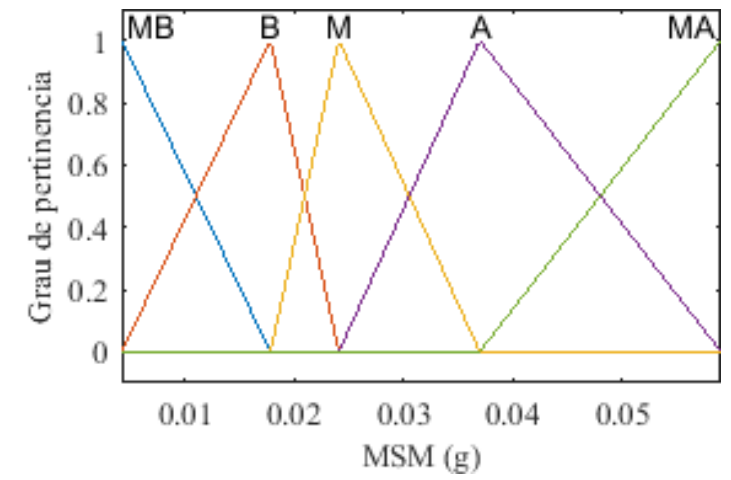

(c)

De acordo com a proposta metodológica de elaboração da base de regras, foi possível gerar as regras utilizada pelo sistema fuzzy em questão para classificar as variáveis de saída de acordo com o tratamento escolhido (Tabela 4). 
Tabela 4. Base de regras do sistema fuzzy.

\begin{tabular}{|c|c|c|c|c|}
\hline \multicolumn{2}{|c|}{ Variáveis de entrada } & \multicolumn{3}{|c|}{ Variáveis de saída } \\
\hline POLÍMERO & IRRIGAÇÃO & CPA & CR & MSM \\
\hline MB & MA & A & A & $M$ \\
\hline $\mathrm{MB}$ & A & M & A & $M$ \\
\hline MB & M & M & A & B \\
\hline MB & B & B & A & B \\
\hline $\mathrm{MB}$ & $\mathrm{MB}$ & B & A & B \\
\hline B & MA & A & A & A \\
\hline B & A & A & A & A \\
\hline B & $\mathrm{M}$ & A & $\mathrm{M}$ & A \\
\hline B & B & $\mathrm{M}$ & $\mathrm{M}$ & $\mathrm{M}$ \\
\hline B & MB & B & B & $\mathrm{M}$ \\
\hline M & MA & A & A & A \\
\hline $\mathrm{M}$ & A & A & A & A \\
\hline M & $\mathrm{M}$ & A & A & $\mathrm{M}$ \\
\hline M & B & $M$ & $M$ & $\mathrm{M}$ \\
\hline M & $\mathrm{MB}$ & B & A & $\mathrm{M}$ \\
\hline A & MA & MA & $\mathrm{M}$ & A \\
\hline A & A & A & A & A \\
\hline A & $\mathrm{M}$ & A & $\mathrm{M}$ & M \\
\hline A & B & $M$ & B & $\mathrm{M}$ \\
\hline A & $\mathrm{MB}$ & B & MB & $\mathrm{MB}$ \\
\hline MA & MA & A & MB & A \\
\hline MA & A & A & $M$ & A \\
\hline MA & M & $\mathrm{M}$ & M & $\mathrm{M}$ \\
\hline MA & B & B & B & B \\
\hline MA & $\mathrm{MB}$ & MB & MB & MB \\
\hline
\end{tabular}

\section{Resultados práticos}

Utilizando os métodos de inferência e defuzzyficação adotados, obtém-se os gráficos tridimensionais e seus respectivos mapas de contorno das variáveis de saída como solução do sistema (Figura 6). 
Figura 6. Gráficos tridimensionais e os respectivos mapas de contorno representando o desenvolvimento das variáveis de saída do SBRF. (a) Comprimento da parte aérea, (b) Comprimento da raiz e (c) Massa seca da muda.
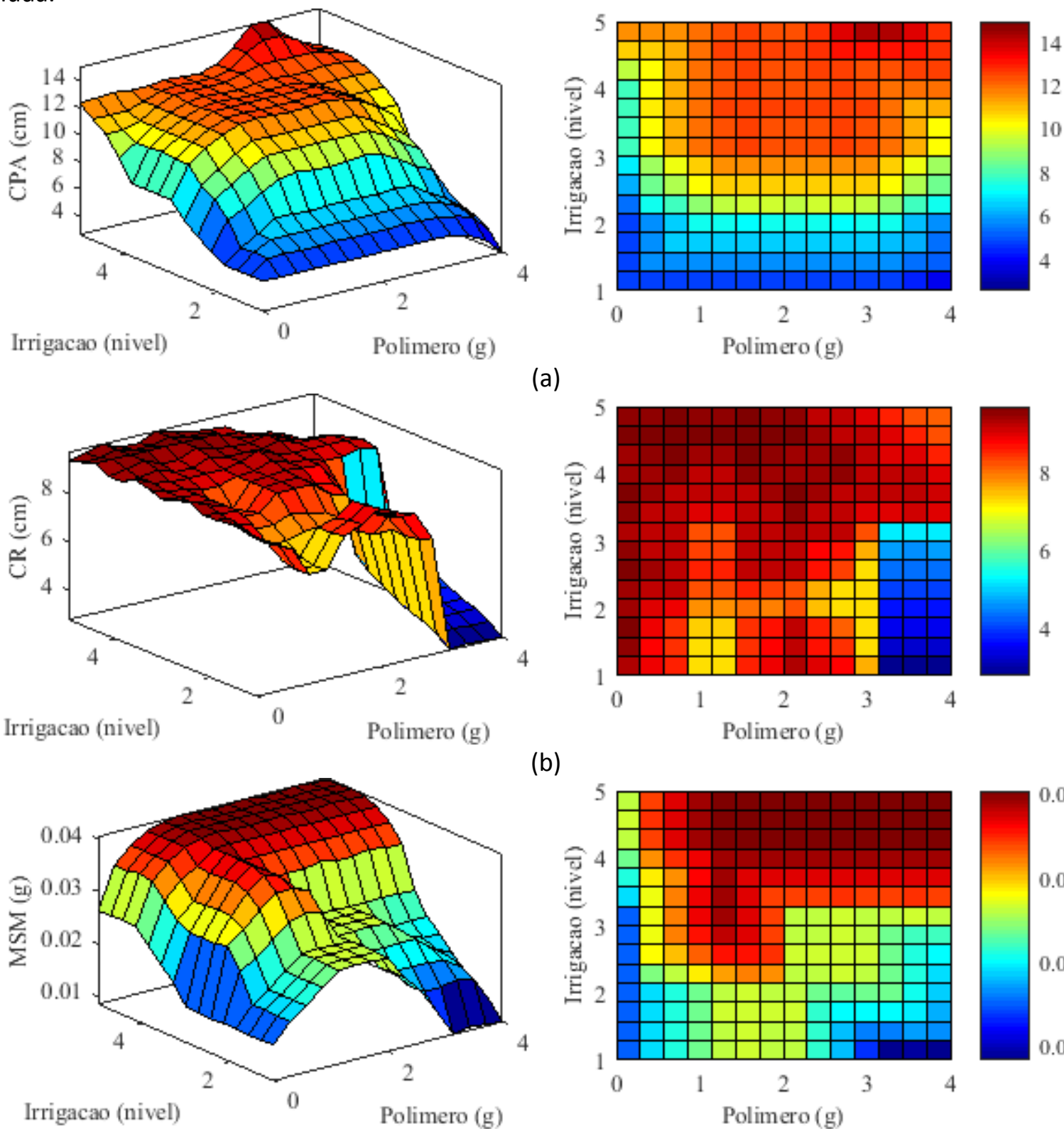

(b)

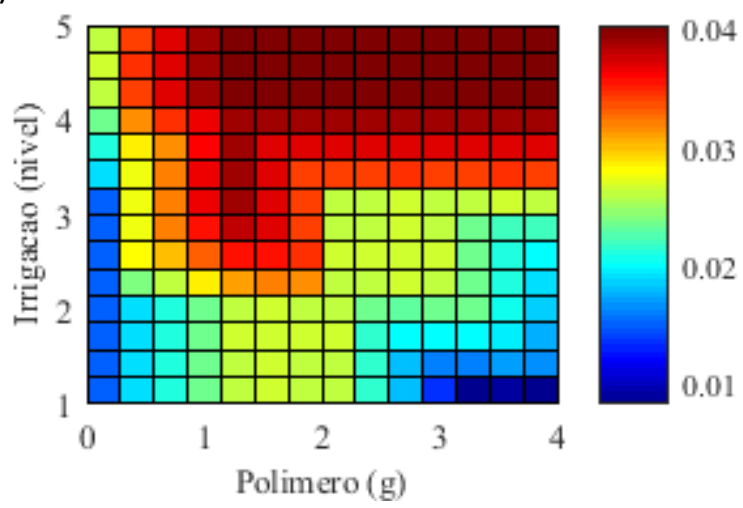

(c)

Em relação ao comprimento da parte aérea da muda (CPA) (Figura 6a), verificou-se que os maiores valores foram obtidos quando as mudas tiveram maior disponibilidade de água devido à irrigação, e que ocorreu um ponto de máximo para o nível de irrigação 5 e adição de polímero $3 \mathrm{~g}$. Também é possível observar que para os níveis de irrigação 1 e 2 , independente da dosagem de polímeros, ocorreu um baixo desenvolvimento da altura das mudas. Para Mews et al. (2015) e Menegatti et al. (2017), a utilização de polímero incorporado ao substrato de produção das mudas ipê-amarelo e guatambu, respectivamente, influenciou positivamente no crescimento das mudas, já Tittonel et al. (2002),

afirmam que a adição do polímero ao substrato permitiu melhorar a precocidade, uniformidade e um aumento na taxa de crescimento de mudas de pimenta.

Já para o comprimento da raiz (CR) (Figura 6b), observa-se que para os níveis de irrigação 4 ou 5 , existe um bom desenvolvimento de seu comprimento. Além disso, verifica-se este mesmo desempenho para o nível de irrigação 1 , o que ocorreu, possivelmente, devido à necessidade hídrica e de nutrientes da planta. Nota-se também que, a adição de polímeros que causou efeito para os menores níveis de irrigação foi a dosagem $2 \mathrm{~g}$, pois houve um maior valor da massa seca da muda. Em relação a esta variável, 
Hafle et al. (2008), notaram que a dose $4,5 \mathrm{~g} \mathrm{~L}^{-1}$ de polímero mostrou-se mais eficiente no enraizamento das estacas do maracujazeiro-doce, porém em doses mais elevadas, ocorreu efeitos negativos para o enraizamento e desenvolvimento das mudas.

$\mathrm{Na}$ análise da massa seca da muda (MSM) (Figura $6 \mathrm{c}$ ), constatou-se que os maiores valores ocorreram para níveis de irrigação 4 e 5 , e com adição de polímeros entre 1 e $4 \mathrm{~g}$. Nesta região, as plantas apresentaram também os maiores valores de altura. Já em níveis menores de irrigação, os maiores valores para a variável MSM se deram com a adição de polímeros entre 1 e 2 g. Para Marques e Bastos (2010), o uso de doses de polímeros interferiu na massa seca da parte aérea de mudas de pimentão por promover maior número de folhas. Navroski et al. (2015), verificaram que houve incremento na massa seca de mudas de Eucalyptus dunnii ao testar diferentes doses de polímeros, além de constatar a redução do uso de irrigação para uma dose de 3 $\mathrm{g} \mathrm{L}^{-1}$ misturado ao substrato. Já Batista et al. (2016) constataram que a dose de $4,5 \mathrm{~g} \mathrm{~L}^{-1}$ de polímero proporcionou maiores quantidades de massa seca em mudas de cana-de-açúcar.

\section{CONCLUSÕES}

A análise dos resultados do modelo fuzzy permite concluir que, para níveis de irrigação inferiores ao máximo (irrigação diária), a utilização de 2 gramas de polímeros remete às melhores condições das variáveis comprimento da parte aérea, comprimento da raiz e massa seca da muda.

\section{REFERÊNCIAS}

ALBUQUERQUE, P. E. P.; ANDRADE, C. L. T. Planilha eletrônica para a programação da irrigação de culturas anuais. Sete Lagoas: Embrapa, 2001. (Circular técnica; 10). Disponível em:

https://ainfo.cnptia.embrapa.br/digital/bitstream /CNPMS/13765/1/Circ_10.pdf

ALVARENGA, M. A. R. Tomate: produção em campo, em casa-de-vegetação e em hidroponia.. Lavras: UFLA, 2004.

BATISTA, L. E.; BULGARELLI, M. I.; RUIZ, J. G. C. L.; MARTINELLI, P. R. P. Uso de gel hidroabsorvente no plantio de mudas de cana-de-açúcar. In: CONGRESSO DE INOVAÇÃO, CIÊNCIA E TECNOLOGIA DO IFSP. Anais... Matão, 2016.
CARVALHO, R. P.; CRUZ, M. C. M.; MARTINS, L. M. Frequência de irrigação utilizando polímero hidroabsorvente na produção de mudas de maracujazeiro-amarelo. Rev. Bras. Frutic., Jaboticabal, v.35, n.2, p.518-526, 2013. https://doi.org/10.1590/S0100$\underline{29452013000200022}$

CREMASCO, C. P. Aplicação da lógica fuzzy para avaliação do faturamento do consumo de energia elétrica e demanda de uma empresa de avicultura de postura. 2008. Tese (Doutorado em Agronomia) - FCA/UNESP, Botucatu-SP, 2008.

CREMASCO, C. P.; GABRIEL FILHO, L. R. A.; CATANEO, A. Metodologia de determinação de funções de pertinência de controladores fuzzy para a avaliação energética de empresas de avicultura de postura. Revista Energia na Agricultura, v.25, p.21-39, 2010. https://doi.org/10.17224/EnergAgric.2010v25n1p 21-39

GABRIEL FILHO, L. R. A. Sistemas Fuzzy aplicados nas Ciências Agrárias. 2015. 258p. Tese (LivreDocência em Matemática Aplicada e Computacional) - FCE/UNESP, Tupã-SP, 2015.

GABRIEL FILHO, L. R. A.; CREMASCO, C. P.; PUTTI, F. F.; CHACUR, M. G. M. Application of fuzzy logic for the evaluation of livestock slaughtering. Engenharia Agrícola, v.31, n.4, p.813-825, 2011. https://doi.org/10.1590/S0100$\underline{69162011000400019}$

HAFLER, O. M.; CRUZ, M. C. M.; RAMOS, J. D.; RAMOS, P. S.; SANTOS, V. A. Produção de mudas de maracujazeiro-doce através da estaquia utilizando polímero hidrorretentor. Revista Brasileira de Ciências Agrárias, v.3, n.3, p.232236, 2008.

https://doi.org/10.5039/agraria.v3i3a292

LECIEJEWSKI, P. The effect of hydrogel additives on the water retention curve of sandy soil from forest nursery in Julinek. Journal Water Land Development, v.13a, p.239-247, 2009. Disponível em:

http://yadda.icm.edu.pl/yadda/element/bwmeta 1.element.baztech-article-BAT9-0018-0025

MAMDANI, E. H.; ASSILIAN, S. An experiment in linguistic synthesis with a fuzzy logic controller. 
International Journal Man-Machine Studies, v.7, p.1-13, 1975. https://doi.org/10.1016/S0020$\underline{7373(75) 80002-2}$

MARIA, A. C. G.; OLIVEIRA, G. S. Produção de mudas de tomate cereja com uso de polímeros e taxas variadas de irrigação. 2017. Trabalho de Conclusão de Curso (Tecnologia em Agronegócio) - Faculdade de Tecnologia de Presidente Prudente, Presidente Prudente-SP, 2017.

MARQUES, P. A. A.; BASTOS, R. O. Usos de diferentes doses de hidrogel para produção de mudas de pimentão. Revista Pesquisa Aplicada \& Agrotecnologia, v.3, n.2, p.53-57, 2010.

MENEGATTI, R. D.; GUOLLO, K.; NAVROSKI, M. C.; POSSENTI, J. C.; SOUZA, A. G. Polímero hidroretentor no substrato para produção de mudas de Aspidosperma parvifolium A. DC. In: SIMPÓSIO DE PROPAGAÇÃO DE PLANTAS E PRODUÇÃO DE MUDAS - INOVAÇÕES EM BUSCA DE QUALIDADE. Anais... Ribeirão Preto, 2017.

MEWS, C. L.; SOUSA, J. R. L.; AZEVEDO, G. T. O. S.; SOUZA, A. M. Efeito do hidrogel e ureia na produção de mudas de Handroanthus ochraceus (Cham.) Mattos. Floresta e Ambiente, Seropédica, v.22, n.1, p.107-116, 2015. http://dx.doi.org/10.1590/2179-8087.080814

MINAMI, K. Produção de mudas de alta qualidade em horticultura. São Paulo, 1995. $128 p$.

MORAES, O. Efeito do uso de polímero hidroretentor no solo sobre o intervalo de irrigação na cultura da alface (Lactuca sativa L.). 2001. 73 f. Tese (Doutorado em Agronomia) Escola Superior de Agricultura Luiz de Queiroz, Piracicaba-SP, 2001.

NAVROSKI, M. C.; ARAÚJO, M. M.; FIOR, C. S.; CUNHA, S. F.; BERGHETTI, A. L. P.; PEREIRA, M. O. Uso de hidrogel possibilita redução da irrigação e melhora o crescimento inicial de mudas de Eucalyptus dunnii Maiden. Scientia Forestalis. Piracicaba, v.43, n.106, p.467-476, 2015.

PUTTI. F. F. Análise dos indicadores biométricos e nutricionais da cultura da alface (Lactuca sativa L.) irrigada com água tratada magneticamente utilizando modelagem fuzzy. 2015. 205f. Tese (Doutorado em
Agronomia/Irrigação e Drenagem) - FCA/UNESP. Botucatu-SP, 2015. Disponível em: http://hdl.handle.net/11449/132147

SEABRA JÚNIOR, S.; GADUM, J.; CARDOSO, I. I. Produção de pepino em função da idade das mudas produzidas em recipientes com diferentes volumes de substrato. Horticultura Brasileira, Brasília, v.22, n.3, p.610-613, jul./set. 2004. http://dx.doi.org/10.1590/S0102-

05362004000300022.

SOUZA, J. L. Tomate para mesa em sistema orgânico. Informe Agropecuário, Belo Horizonte, v.24, n.219, p.109-120, 2003.

TITTONELL, P. A.; GRAZIA, J.; CHIESA, A. Adición de polímeros superabsorbentes en el medio de crecimiento para la producción de plantines de pimiento. Horticultura Brasileira, Brasília, v.20, n.4, p.641-645, 2002. https://doi.org/10.1590/S0102$\underline{05362002000400026}$

VALE, G. F. R.; CARVALHO, S. P.; PAIVA, L. C. Avaliação da eficiência de polímeros hidroretentores no desenvolvimento do cafeeiro em pós-plantio. Coffee Science, v.1, p.7-13, 2006. http://dx.doi.org/10.25186/cs.v1i1.12

VIAIS NETO, D. S. Modelagem fuzzy para avaliação do desenvolvimento do tomate em tensões de água no solo e doses de salinidade na irrigação. 2016, 70f. Tese (Doutorado em Agronomia/Irrigação e Drenagem) - FCA/UNESP. Botucatu-SP, 2016. http://hdl.handle.net/11449/139498

Recebido para publicação em 05/04/2018

Revisado em 28/08/2018

Aceito em 02/09/2018 